\title{
REPRESENTATIONS OF COMPACT LIE GROUPS OF LOW COHOMOGENEITY
}

\author{
FRANCISCO J. GOZZI
}

\begin{abstract}
We survey different tools to classify representations of compact Lie groups according to their cohomogeneity and apply these methods to the case of irreducible representations of cohomogeneity 6,7 and 8 .
\end{abstract}

\section{INTRODUCTION}

A fundamental invariant of a finite-dimensional representation of a compact Lie group is its cohomogeneity, which by definition is the minimal codimension $c$ of its orbits, and also agrees with the topological dimension of the orbit space.

Representations with low cohomogeneity should display interesting geometric and topological properties. Indeed those with the minimum possible value $c=1$ are necessarily irreducible and coincide with the transitive isometric actions on unit spheres; they were probably first explicitly listed by Borel and MontgomerySamelson Bor49, MS43, who in fact classified (effective) transitive smooth actions of compact connected Lie groups on spheres. The cases $c=2$ and $c=3$ were studied by Hsiang-Lawson [HL71] (see also [Str94]), in connection with the construction of minimal submanifolds with large groups of symmetries. They noticed that the maximal connected groups with $c=2$ always act by the isotropy representation of a symmetric space, and the same is true in the irreducible case if $c=3$, up to three exceptions. More generally, representations with $c=2$ are always polar [BCO03, and the irreducible ones with $c=3$ are always taut GT03.

The complexity of representations grows with $c$ and it is therefore natural to consider first irreducible representations. In GL14 irreducible representations with $c=4$ and $c=5$ were classified in connection to certain problems regarding the geometry of orbit spaces. Also, some interesting representations with $c=7$ associated to quaternion-Kahler symmetric spaces naturally appeared in [GG]. The purpose of this paper is to organize, extend and refine the techniques from GL14 to allow for a classification of representations of higher cohomogeneity. In view of the inductive nature of our method, often requiring information about lower cohomogeneity cases, we found useful to do a calculation of some principal isotropy groups. These techniques are finally applied to classify irreducible representations with $6 \leq c \leq 8$ and we obtain:

Theorem. A compact connected Lie group irreducible representation of cohomogeneity 6,7 or 8 is either polar or, else, listed in Tables $I V, \square$ or $\square]$.

2010 Mathematics Subject Classification. 57S15, 22E46.

The author has been supported by the FAPESP fellowship 2014/22568-1. 
This paper is organized as follows. The first section establishes terminology and preliminary considerations, where useful principal isotropy computations and cohomogeneity estimates are given for sums and tensors of representations. The second section represents the core of this work where the available tools are discussed, distinguishing among simple and non-simple Lie groups. Last, the third section sketches the classification for arbitrary cohomogeneity and illustrates the procedure by carrying it through up to cohomogeneity 8 . The main tables are displayed at the end.

The author is indebted to Claudio Gorodski for suggesting this project and for many fruitful discussions, his was the suggestion for the proof of Remark 2.2.

\section{Preliminaries}

Let $\rho: G \rightarrow \mathrm{O}(V)$ be an orthogonal representation of a compact Lie group $G$ on a finite-dimensional Euclidean vector space $V$. The stabilizer of a point $p \in V$ is denoted by $G_{p}$ and referred to as its isotropy group, and the orbit through $p$ is denoted by $G p$. Isotropy subgroups along a $G$-orbit lie in the same conjugacy class. The conjugacy classes of isotropy groups, called isotropy types, give a natural stratification of the orbit space. Moreover, the inclusion of subgroups induces a lattice structure on the set of isotropy types. The Principal Orbit Type Theorem asserts that there is a unique minimal istropy type, called the principal isotropy type; the corresponding orbits are called principal orbits. The union of principal orbits is a open dense subset of $V$, and its projection to the orbit space is a connected, open dense subset called the principal stratum. We will denote a fixed principal isotorpy group by $H$.

The cohomogeneity of a representation equals the codimension of a principal orbit $G / H$ in $V$, and thus it can be computed from $(H)$ from the following equation:

$$
c=c(\rho)=\operatorname{dim}(V)-\operatorname{dim}(G)+\operatorname{dim}(H) .
$$

There is an algorithm to compute a principal isotropy group of a representation $\rho$ based on the fact that the set of isotropy groups of any the slice representation of $\rho$ contain representatives of all isotropy types of $\rho$ [Hsi67]. Namely, take any point $p$ in $V$ which is not fixed by $G$. Then there is a $G_{p}$-invariant decomposition

$$
V=T_{p}(G p) \oplus N_{p}(G p)
$$

where $T_{p}(G p)$ and $N_{p}(G p)$ denote the tangent and normal spaces to $G p$ at $p$, respectively. The slice representation of $\rho$ at $p$ is the action of $G_{p}$ on $N_{p}(G p)$. A principal isotropy group for $\left(G_{p}, N_{p}(G p)\right)$ is a principal isotropy group for $(G, V)$ as well, and we have reduced our calculation to the case of a representation of lower dimensional group and lower dimensional representation space. By dimensional reasons, the algorithm evetually stops when the slice representation is trivial.

In general, the calculation of full isotropy groups can be a delicate matter and in most cases we will only need their isotropy groups which are much easier to obtain. It is useful to note that, by considering only the identity component of the isotropy group at each step of the algorithm, we end up with the identity component of the principal isotropy group.

1.1. Reducible representations. Although we are mostly interested in the case of irreducible representations, reducible ones are bound to appear for instance 
as slice representations at singular points. It is plain that for a $G$-invariant decomposition $V=V_{1} \oplus V_{2}$, a point $p=\left(p_{1}, p_{2}\right)$ with $p_{i} \in V_{i}$ has isotropy group $G_{p}=G_{p_{1}} \cap G_{p_{2}}$; and $G_{p_{i}}$ being a principal isotropy group of $\left(G, V_{i}\right)$ for $i=1,2$ is a necessary but not sufficient condition for $G_{p}$ to be a principal isotropy group of $(G, V)$. In general, it follows from the discussion above about slice representations that a principal isotropy group of $(G, V)$ coincides with a principal isotropy group of $\left(H_{1}, V_{2}\right)$, where $H_{1}$ is a principal isotropy group of $\left(G, V_{1}\right)$.

For example, given two copies of a non-trivial representation $(G, V)$, note that a principal isotropy group $H$ acts reducibly on $V$, where $V^{H}$ is a proper subspace of dimension equal to $c(G, V)$, and that $c(G, 2 V)=c(G, V)+c(H, V)$ and $c(H, V)=$ $c(G, V)+c\left(H, T_{[H]} G / H\right)$, so

$$
c(G, 2 V)=2 c(G, V)+c\left(H, T_{[H]} G / H\right) \geq 2 c(G, V)+1 .
$$

1.2. Real tensor products. Let $\rho: G \rightarrow \mathrm{O}(V)$ be a representation of the form $\rho=\rho_{1} \otimes \rho_{2}$, where $G=G_{1} \times G_{2}, V=V_{1} \otimes_{\mathbb{R}} V_{2}$ and $\rho_{i}: V_{i} \rightarrow \mathrm{O}\left(V_{i}\right)$ for $i=1,2$. We consider the slice representation at a pure tensor $p=p_{1} \otimes p_{2}$, where $H_{i}=G_{p_{i}}$ is a principal isotropy group for $i=1,2$.

Note first that, for the identity components, $G_{p}^{0}=H_{1}^{0} \times H_{2}^{0}$. Furthermore, since the Lie algebra of $G$ acts on $V_{1} \otimes V_{2}$ by derivations, the tangent space

$$
T_{p}(G p)=\left(T_{p_{1}}\left(G_{1} p_{1}\right) \otimes p_{2}\right) \oplus\left(p_{1} \otimes T_{p_{2}}\left(G_{2} p_{2}\right)\right) .
$$

The slice representation has $\mathbb{R} p$ as a trivial summand, so it is natural to consider the reduced normal space $\tilde{N}_{p}(G p)=N_{p}(G \cdot v) \ominus \mathbb{R} p$, and then

$$
\tilde{N}_{p}(G p)=\left(\tilde{N}_{p_{1}}\left(G_{1} p_{1}\right) \otimes p_{2}\right) \oplus\left(p_{1} \otimes \tilde{N}_{p_{2}}\left(G_{2} p_{2}\right)\right) \oplus\left(p_{1}^{\perp} \otimes p_{2}^{\perp}\right) .
$$

The group $H_{1}^{0} \times H_{2}^{0}$ acts trivially on the first two summands of the right hand-side of (1.3) leading to

$$
c(G, V)=c\left(G_{1}, V_{1}\right)+c\left(G_{2}, V_{2}\right)-1+c\left(H_{1}^{0} \times H_{2}^{0}, p_{1}^{\perp} \otimes p_{2}^{\perp}\right) .
$$

It is useful to discuss further the last term on the right hand-side of (1.4) In some particular cases. Write $T_{p_{i}}\left(G_{i} p_{i}\right)=U_{i}, \tilde{N}_{p_{i}}\left(G p_{i}\right)=\tilde{N}_{i}$ and $c\left(G_{i}, V_{i}\right)=c_{i}$. If $c_{1}=1$, then $v_{1}^{\perp} \otimes v_{2}^{\perp}$ equals $\left(U_{1} \otimes U_{2}\right) \oplus\left(U_{1} \otimes \tilde{N}_{2}\right)$. The action of $H_{1}^{0} \times H_{2}^{0}$ on the second summand equals to $c_{2}-1$ copies of $\left(H_{1}, U_{1}\right)$, whose principal isotropy group is therefore of the form $K_{1} \times H_{2}^{0}$ for a subgroup $K_{1}$ of $H_{1}^{0}$. We finally obtain that

$$
c(G, V)=c_{2}+c\left(H_{1}^{0},\left(c_{2}-1\right) U_{1}\right)+c\left(K_{1} \times H_{2}^{0}, U_{1} \otimes U_{2}\right) .
$$

\section{MAIN}

2.1. Simple Lie Groups. A representation of a compact simple Lie group $G$ with a certain bound on the cohomogeneity either satisfies the condition of low degree defined by A. Kollross in Kol02 which leads to a concrete list of candidates, or $G$ has bounded dimension. In the latter case, we can resort to a case by case analysis of the finitely many possible groups making use of basic representation theory. For a fixed maximal torus of $G$ and an ordering of the roots. Then we have the simple roots by $\alpha_{1}, \ldots, \alpha_{r}$ and the fundamental weights $\lambda_{1}, \ldots, \lambda_{r}$, and the degree of a 
complex irreducible representation is a strongly monotonous function of the $\lambda_{i}$, as observed by A. L. Onishchik in Oni04. By making use of the basic inequality

$$
c(\rho) \geq \operatorname{deg}(\rho)-\operatorname{dim}(G),
$$

we may therefore bound the degree for each possible group and obtain a finite list of representations.

The problem of determining the principal isotropy type and, hence, the exact cohomogeneity of a given $n$-dimensional representation is completely solved in the case of compact simple Lie groups in [HH70. It is interesting to reformulate their result as follows.

Proposition 2.1. An irreducible representation of a compact connected simple Lie group with non-trivial principal isotropy type is either polar or a simple factor of the isotropy representation associated to a symmetric space of Hermitian or quaternionKähler type.

We recall that a representation is called polar if there exists a subspace, called a section, meeting all orbits and always orthogonally. It follows from the classification of Dadok [Dad85] that a polar representation is orbit-equivalent to (i.e. has the same orbits as) the isotropy representation of a symmetric space, and indeed the maximal groups in each orbit-equivalence class act by isotropy representations of symmetric spaces. It turns out that the only irreducible polar representations with trivial principal isotropy groups are the standard actions of $\mathrm{SO}(2)$ on $\mathbb{R}^{2}$ and of $\mathrm{SU}(2)$ on $\mathbb{C}^{2}$ GL15a.

A representation $(G, V)$ with non-trivial principal isotropy group $H$ admits the so called core reduction, or Luna-Richardson-Straume reduction in the linear case, namely, the quotient group $N_{G}(H) / H$ acts effectively on the fixed point set $V^{H}$ with orbit space isometric to the orbit space $V / G$. More generally, a reduction of $(G, V)$ is a representation $(K, W)$ with $\operatorname{dim} K<\operatorname{dim} G$ and orbit space isometric to $V / G$ GL14. In the special case $N_{G}(H) / H$ is finite, the representation $(G, V)$ is called asystatic, and it is automatically polar with $V^{H}$ as a section ( AA93, see also [GZ12]). Remarkably, every polar representations admits an orbit-equivalent finite extension to an asystatic representation of a disconnected Lie group, as observed in Str94 (see also GK16]). The non-polar representations satisfying the conditions in Proposition 2.1 are given by the restriction to the non-U(1) factor (resp. non-Sp(1) factor) of the isotropy representation of Hermitian (resp. quaternion-Kähler symmetric space). They admit reductions to tori (resp. Sp $(1)^{3}$-subgroups), and were investigated in [GL15b] and [GG].

We shall refer to the Tables in Subsection 12.8 in GL14 listing polar and nonpolar irreducible representations of compact connected simple Lie groups of cohomogeneity up to 8 . In addition, we extend those lists to include cohomogeneity 9 in Table 2.1

Remark 2.2. At this juncture, it is worth commenting on the half-spin representation of $\operatorname{Spin}(14)$ on $\mathbb{C}^{64}$ that appears on [HH70, Table A]. It can be shown to have trivial principal isotropy group by an argument similar to Example 1 in that paper. Indeed, if $p$ is a highest weight vector, it is easy to see that $G_{p}^{0}=\operatorname{SU}(7)$. Moreover, from the theory of parabolic subgroups, it is known that the normalizer of the line $\mathbb{R} p$ is $\mathrm{U}(7)$. Finally, let $T$ and $\tilde{T}$ be maximal tori in $\mathrm{SU}(7), \mathrm{U}(7)$, respectively. It is easy to see that no non-trivial elements of $\tilde{T} / T$ fix $p$ and, thus, 


\begin{tabular}{|ccc|}
\hline$G$ & $V$ & Polar? \\
\hline $\mathrm{SO}(10)$ & $S_{0}^{2} \mathbb{R}^{10}$ & Yes \\
$\mathrm{Sp}(10)$ & $\Lambda^{2} \mathbb{C}^{20} \ominus \mathbb{C}$ & Yes \\
$\mathrm{SU}(10)$ & Adjoint & Yes \\
$\mathrm{SO}(18)$ & Adjoint & Yes \\
$\mathrm{SO}(19)$ & Adjoint & Yes \\
$\mathrm{Sp}(10)$ & Adjoint & Yes \\
$\mathrm{SU}(19)$ & Adjoint & Yes \\
$\mathrm{SU}(2)$ & $\mathbb{C}^{6}$ & No \\
$\mathrm{SU}(8)$ & $S^{2} \mathbb{C}^{8}$ & No \\
$\mathrm{Spin}(11)$ & $\mathbb{H}^{16}$ & No \\
\hline
\end{tabular}

TABLE I. Irreducible representations of cohomogeneity 9 of simple Lie groups.

$G_{p}=\mathrm{SU}(7)$ is the full isotropy subgroup. Furthermore, its slice representation is given by $\mathbb{R} \oplus \mathbb{C}^{7} \oplus \Lambda^{3} \mathbb{C}^{7}$ which has trivial principal isotropy.

2.2. Non-simple Lie groups. For the case of real representations of non-simple compact Lie groups, it is easier to start with complex representations since they have a simple description in terms of tensor products of complex irreducible representations of the factors of $G$.

A complex representation $\pi$ is called of real type if it comes from a representation on a real vector space by extension of scalars, and it is called of quaternionic type if it comes from a representation on a quaternionic (right-)vector space by restriction of scalars. If $\pi$ is neither of real type nor of quaternionic type, we say that $\pi$ is of complex type. If $\pi$ is irreducible, then it is exactly of one of those types.

Now it is known that the finite-dimensional real irreducible representations $\rho$ of $G$ fall into one of the following disjoint classes:

(a) the complexification $\rho^{c}$ is irreducible and $\rho^{c}=\pi$ is a complex representation of real type;

(b) the complexification $\rho^{c}$ is reducible and $\rho^{c}=\pi \oplus \pi$ where $\pi$ is a complex irreducible representation of quaternionic type;

(c) the complexification $\rho^{c}$ is reducible and $\rho^{c}=\pi \oplus \pi^{*}$ where $\pi$ is a complex irreducible representation of complex type and $\pi^{*}$ is not equivalent to $\pi$ (where $\pi^{*}$ denotes the dual representation of $\pi$ ).

The relation between $\rho$ and $\pi$ is that $\rho$ is a real form of $\pi$ in the first case $\left(\rho^{c}=\pi\right.$ and $\left.\rho=[\pi]_{\mathbb{R}}\right)$. In the other two cases $\rho$ is $\pi$ viewed as a real representation, its "realification". We shall call $\rho$ of real, quaternionic or complex type according to whether the associated $\pi$ is of real, quaternionic or complex type. Note also that $\pi$ is self-dual precisely in the first two cases.

A real (resp. quaternionic) structure for $\pi$ can be equivalently given by an equivariant conjugate-linear endomorphism $\epsilon$ such that $\epsilon^{2}=1$ (resp. $\left.\epsilon^{2}=-1\right)$. It follows that if $\epsilon_{i}$ is such a structure for $\pi_{i}$ then $\epsilon_{1} \otimes \epsilon_{2}$ is such a structure for $\pi_{1} \otimes_{\mathbb{C}} \pi_{2}$, and $\pi_{1} \otimes_{\mathbb{C}} \pi_{2}$ is of complex type whenever one of the factor is. Table II summarizes our discussion. 


\begin{tabular}{l|lll} 
& $r$ & $q$ & $c$ \\
\hline$r$ & $r$ & $\cdot$ & $\cdot$ \\
$q$ & $q$ & $r$ & $\cdot$ \\
$c$ & $c$ & $c$ & $c$
\end{tabular}

TABLE II. The type of $\rho_{1} \otimes_{\mathbb{C}} \rho_{2}$ in terms of $\rho_{1}$ and $\rho_{2}$.

Remark 2.3. We collect some useful remarks about real forms and realifications of tensor products of complex representations that will allow us to use ideas from Section 1.2

(i) If $\pi_{1}$ and $\pi_{2}$ are of are of real type, a real form of $\pi=\pi \otimes_{\mathbb{C}} \pi_{2}$ can be obtained as $[\pi]_{\mathbb{R}}=\left[\pi_{1}\right]_{\mathbb{R}} \otimes_{\mathbb{R}}\left[\pi_{2}\right]_{\mathbb{R}}$.

(ii) If $\pi_{1}$ is of real type and $\pi_{2}$ is of complex or quaternionic type, the realification of $\pi=\pi_{1} \otimes_{\mathbb{C}} \pi_{2}$ can be described as $\pi^{r}=\left[\pi_{1}\right]_{\mathbb{R}} \otimes_{\mathbb{R}} \pi_{2}^{r}$.

(iii) If $\pi_{1}$ and $\pi_{2}$ are of quaternionic type, a real form of $\pi=\pi_{1} \otimes_{\mathbb{C}} \pi_{2}$ can be obtained as $[\pi]_{\mathbb{R}}=\pi_{1} \otimes_{\mathbb{H}} \pi_{2}$. We refer to [GL14, Subsection 12.3] for an explanation about quaternionic tensor products.

(iv) When $\pi_{1}$ is of complex type and $\pi_{2}$ is not of real type, we must deal directly with the realification of $\pi_{1} \otimes_{\mathbb{C}} \pi_{2}$.

A representation which is given as a real, complex or quaternionic tensor product is naturally a restriction of $\left(\mathrm{SO}(m) \times \mathrm{SO}(n), \mathbb{R}^{m} \otimes_{\mathbb{R}} \mathbb{R}^{n}\right),\left(\mathrm{U}(m) \times \mathrm{U}(n), \mathbb{C}^{m} \otimes_{\mathbb{C}} \mathbb{C}^{n}\right)$ or $\left(\mathrm{Sp}(m) \times \mathrm{Sp}(n), \mathbb{H}^{m} \otimes_{\mathbb{H}} \mathbb{H}^{n}\right)$, respectively. Hence, we obtain a lower bound for the cohomogeneity, namely, the minimum between $m$ and $n$. For simplicity, below we only list the identity components of the corresponding principal isotropy groups (see for instance [HH70]).

Lemma 2.4. For a representation $(G, V)$, let $H$ denote a principal isotropy group. Assume $m \geq n$. Then:

(1) For $\left(\mathrm{SO}(m) \times \mathrm{SO}(n), \mathbb{R}^{m} \otimes_{\mathbb{R}} \mathbb{R}^{n}\right)$, we have $H^{0}=\mathrm{SO}(m-n)$.

(2) For $\left(\mathrm{U}(m) \times \mathrm{U}(n), \mathbb{C}^{m} \otimes_{\mathbb{C}} \mathbb{C}^{n}\right)$, we have $H^{0}=\mathrm{U}(m-n) \times \mathrm{U}(1)^{n}$.

(3) For $\left(\mathrm{Sp}(m) \times \mathrm{Sp}(n), \mathbb{H}^{m} \otimes_{\mathbb{H}} \mathbb{H}^{n}\right)$, we have $H^{0}=\mathrm{Sp}(m-n) \times \mathrm{Sp}(1)^{n}$.

With a view toward complex tensor products of mixed type, we state the following result whose proof is straightforward based on the algorithm described in Section 1. From the information about principal isotropy groups we can of course deduce the cohomogeneity of the involved representations.

Lemma 2.5. For a representation $(G, V)$, let $H$ denote a principal isotropy group. Then:

(1) For $\left(\mathrm{SO}(m) \times \mathrm{U}(n), \mathbb{R}^{m} \otimes_{\mathbb{R}} \mathbb{C}^{n}\right)$ we have

$$
H^{0}= \begin{cases}\mathrm{SO}(m-2 n) & m>2 n+1, \\ \mathrm{U}(n-m) & n>m,\end{cases}
$$

otherwise it is trivial.

(2) For $\left(\mathrm{SO}(m) \times \mathrm{Sp}(n), \mathbb{R}^{m} \otimes_{\mathbb{R}} \mathbb{H}^{n}\right)$, we have

$$
H^{0}= \begin{cases}\mathrm{SO}(m-4 n) & \text { if } m>4 n+1 \geq 5 ; \\ \mathrm{Sp}(n-m) & \text { if } n>m \geq 3 ;\end{cases}
$$

otherwise it is trivial. 
(3) For $\left(\mathrm{SO}(m) \times \mathrm{Sp}(1) \mathrm{Sp}(n), \mathbb{R}^{m} \otimes_{\mathbb{R}} \mathbb{R}^{4 n}\right)$,

$$
H^{0}= \begin{cases}\mathrm{SO}(m-4 n) & m>4 n+1, \\ \mathrm{Sp}(n-m) & n>m \geq 3,\end{cases}
$$

otherwise it is trivial, as long as $m \geq 3$. For the case $m=2$ and $n \geq 2$ we have that $H^{0}=\{1\} \times \mathrm{U}(1) \operatorname{Sp}(n-2)$.

(4) For $\left(\mathrm{SO}(m) \times \mathrm{U}(1) \mathrm{Sp}(n), \mathbb{R}^{m} \otimes_{\mathbb{R}} \mathbb{C}^{2 n}\right)$ we have

$$
H^{0}= \begin{cases}\mathrm{SO}(m-4 n) & m>4 n \\ \mathrm{Sp}(n-m) & n \geq m\end{cases}
$$

otherwise it is trivial.

(5) For $\left(\mathrm{U}(m) \times \mathrm{Sp}(1) \mathrm{Sp}(n), \mathbb{C}^{m} \otimes_{\mathbb{R}} \mathbb{R}^{4 n}\right)$ we have

$$
H^{0}= \begin{cases}\mathrm{U}(m-4 n) & m \geq 4 n, \\ \mathrm{Sp}(n-2 m) & n \geq 2 m,\end{cases}
$$

otherwise it is trivial, assuming $n \geq 2$.

(6) For $\left(\mathrm{Sp}(1) \mathrm{Sp}(m) \times \mathrm{Sp}(1) \mathrm{Sp}(n), \mathbb{R}^{4 m} \otimes_{\mathbb{R}} \mathbb{R}^{4 n}\right)$ we have

$$
H^{0}=\mathrm{Sp}(m-4 n)
$$

if $m>4 n+1$, otherwise it is trivial.

(7) For $\left(\mathrm{U}(m) \times \mathrm{Sp}(n), \mathbb{C}^{m} \otimes_{\mathbb{C}} \mathbb{H}^{n}\right)$, we have

$$
H^{0}= \begin{cases}\mathrm{U}(m-2 n) & \text { if } m \geq n+1 \geq 2 ; \\ \mathrm{Sp}(n-m) & \text { if } n \geq m \geq 3 ; \\ \mathrm{U}(1) \times \mathrm{U}(m-2) & \text { if } m \geq 2 \text { and } n=1 ; \\ \mathrm{U}(1) \times \mathrm{Sp}(n-m) & \text { if } n \geq m \text { and } 2 \geq m \geq 1 ;\end{cases}
$$

otherwise it is trivial.

(8) For $\left(\mathrm{Sp}(m) \times \mathrm{U}(1) \mathrm{Sp}(n), \mathbb{H}^{m} \otimes_{\mathbb{C}} \mathbb{C}^{2 n}\right)$, we can assume $m \geq n \geq 2$, then $H^{0}=\mathrm{Sp}(m-2 n)$ if $m>2 n$ or, else, it is trivial.

For each family of representations listed in Lemma 2.5 with fixed $m$ (resp. $n$ ), one notes that the cohomogeneity is asymptotically constant on $n$ (resp. $m$ ). This can be deduced in general from the following useful result.

Lemma 2.6 (Monotonicity Lemma GL14]). Let $\rho(n)$ be the $\mathbb{F}$-tensor product of a fixed real representation of $\mathbb{F}$-type with $\mathrm{SO}(n), \mathrm{U}(n)$ or $\mathrm{Sp}(n)$ according to whether $\mathbb{F}=\mathbb{R}, \mathbb{C}$ or $\mathbb{H}$. Then the cohomogeneity of $\rho(n)$ is a non-decreasing function of $n$.

Corollary 2.7. The cohomogeneity of any given representation tensored with one of the standard vector representations: $\left(\mathrm{SO}(n), \mathbb{R}^{n}\right),\left(\mathrm{U}(n), \mathbb{C}^{n}\right)$ or $\left(\mathrm{Sp}(n), \mathbb{H}^{n}\right)$, is asymptotically constant on $n$.

Proof. In case $\mathbb{F}=\mathbb{R}$, assume the real dimension of a given representation is $k$. For $n>k$, the principal isotropy group of $\rho(n)$ contains a normal subgroup of type $\mathrm{SO}(n-k)$, which yields an upper bound on the cohomogeneity. Lemma 2.6 then implies that the cohomogeneity stabilizes, as wished. The other cases are analogous. 


\section{Classification}

Let us sketch a general plan for the classification of irreducible representations of compact connected Lie group with bounded cohomogeneity. We shall then carry this procedure up to cohomogeneity $c \leq 8$.

First, as discussed in Section 2.1 we may determine the irreducible representations of simple Lie groups up to a given cohomogeneity. This task is also an auxiliary one for the cases to come and, hence, we shall see that it suffices to list them up to cohomogeneity $c+3$.

On a second step, we consider those representations that can be expressed as a real tensor product, see Remark 2.3. Though a great deal of cases is to be considered here we have the useful formula (1.4) at our disposal. Observe that both the two factors involved in the tensor have strictly smaller cohomogeneity and are, thus, inductively known.

Third, we have the case of the quaternionic tensor product of two irreducible representations of quaternionic type. Notice that we may assume the group to be a product of two simple factors for, otherwise, we would be able to express the outcome representation as a suitable real tensor product, already considered. Moreover, a standard application of the Monotonicity Lemma 2.6 shows that each (simple) factor has cohomogeneity at most $c+3$. In the following classification up to cohomogeneity 8 we consider more cases in this step and, as a result, we need only list simple Lie group representations up to cohomogeneity 9 .

Finally, the remaining cases are real representations underlying complex tensor products, again after Remark 2.3. These representations split completely as a complex tensor product of its simple factors and, thus, give a subrepresentation of $\mathrm{U}\left(n_{1}\right) \otimes \mathrm{SU}\left(n_{2}\right) \otimes \cdots \mathrm{SU}\left(n_{k}\right)$. Monotonicity can be used to give an upper bound on the number " $k$ " of factors by estimating the cohomogeneity of the initial case $\left(n_{1}, n_{2}, \ldots, n_{k}\right)=(2,3, \ldots, 3)$. Here, once more, we avoid the case of two quaternionic SU(2) factors which leads to a reducible representation. Furthermore, each simple factor has cohomogeneity at most $c+1$.

We emphasize that this is an inductive procedure, though at each step one is required to compute or estimate the cohomogeneity of finite many (possibly infinite) families of representations.

We illustrate the procedure in carrying out the classification of irreducible representations of compact connected Lie groups of cohomogeneity 6,7 or 8 .

3.1. Real tensor products. Let us consider $\rho=\rho_{1} \otimes_{\mathbb{R}} \rho_{2}$ as a restriction of the representation $\mathrm{SO}(m) \otimes_{\mathbb{R}} \mathrm{SO}(n)$, where $\rho_{1}$ and $\rho_{2}$ are real irreducible representations, and assume $6 \leq c(\rho) \leq 8$.

3.1.1. Assume first $m=2$ so that $\rho_{1}=\left(\mathrm{SO}(2), \mathbb{R}^{2}\right)$. Then the second factor $\rho_{2}$ must be of real type for otherwise $\rho$ would be reducible. Formula (1.5) gives

$$
c(\rho)=2 c\left(\rho_{2}\right)-1+c\left(H_{2}, U_{2}\right) \geq 2 c\left(\rho_{2}\right) .
$$

Now $c(\rho) \leq 8$ implies that $c\left(\rho_{2}\right) \leq 4$. Let us run through the possible cases.

First, we cannot have $c\left(\rho_{2}\right)=4$ for otherwise $c\left(H_{2}, U_{2}\right) \geq 3$ which is impossible. In fact, this is clear if $\rho_{2}$ is a polar representation since in this case its principal orbits are irreducible isoparametric submanifolds admitting at least three curvature distributions [BCO03] which provides an $H_{2}$-invariant decomposition of $U_{2}$ with at 
least three components. If $\rho_{2}$ is non-polar, then by [GL14, Table 1] it is $\left(\mathrm{SO}(3), \mathbb{R}^{7}\right)$ or $\left(\mathrm{SO}(3) \times \mathrm{G}_{2}, \mathbb{R}^{3} \otimes_{\mathbb{R}} \mathbb{R}^{7}\right)$; in both cases $H_{2}$ is finite and $c\left(H_{2}, U_{2}\right) \geq 3$ follows.

Second, the case $c\left(\rho_{2}\right)=3$ is discarded as well, by improving the previous estimate to $c\left(H_{2}, U_{2}\right) \geq 5$. In fact, if $\rho_{2}$ is not polar, by classification we know that $\rho_{2}=\left(\operatorname{Sp}(1) \times \operatorname{Sp}(n), \mathbb{H}^{2} \otimes_{\mathbb{H}} \mathbb{H}^{n}\right)$ [HL71], and it follows from results in GT00, that $c\left(H_{2}, U_{2}\right) \geq 9$. If not, polar candidates can be discarded by estimating the value of $c\left(H_{2}, U_{2}\right)$ directly, since their principal isotropy algebras are known. In the case of families of s-representations associated to the orthogonal and quaternionic grassmannians it is enough to estimate the cohomogeneity of the initial cases and apply the Monotonicity Lemma.

Third, if $c\left(\rho_{2}\right)=2$ then $\rho_{2}$ is polar and $c\left(H_{2}, U_{2}\right) \leq 5$. A principal orbit is isoparametric and we use their classification to list the possibilities in Table III, we obtain examples corresponding to the first four cases therein.

\begin{tabular}{|cc|ccc|}
\hline$G_{2}$ & $V_{2}$ & $H_{2}$ & $U_{2}$ & $c\left(H_{2}, U_{2}\right)$ \\
\hline \hline $\mathrm{SO}(3)$ & $\mathbb{R}^{5}$ & $\{1\}$ & $3 \mathbb{R}$ & 3 \\
$\mathrm{Sp}(3)$ & {$\left[\Lambda^{2} \mathbb{C}^{6} \ominus \mathbb{C}\right]_{\mathbb{R}}$} & $\mathrm{Sp}(1)^{3}$ & $3 \mathbb{H}$ & 3 \\
$\mathrm{SU}(3)$ & $\mathfrak{s u}(3) \cong \mathbb{R}^{8}$ & $\mathrm{~T}^{2}$ & $3 \mathbb{C}$ & 4 \\
$\mathrm{~F}_{4}$ & $\mathbb{R}^{26}$ & $\mathrm{Spin}^{26}(8)$ & $\mathbb{R}_{0}^{8} \oplus \mathbb{R}_{+}^{8} \oplus \mathbb{R}_{-}^{8}$ & 4 \\
$\mathrm{Sp}(2)$ & $\mathfrak{s p}(2) \cong \mathbb{R}^{10}$ & $\mathrm{~T}^{2}$ & $4 \mathbb{C}$ & 6 \\
$\mathrm{Sp}(2) \times \mathrm{Sp}(n)$ & $\mathbb{H}^{2} \otimes_{\mathbb{H}} \mathbb{H}^{n}$ & $\mathrm{Sp}(1)^{2} \mathrm{Sp}(n-2)$ & $2\left[\mathbb{H}^{n-2} \oplus \mathbb{R}^{3} \oplus \mathbb{H}\right]$ & $>6$ \\
$\mathrm{SO}(4)$ & $\mathbb{R}^{8}$ & $\mathbb{Z}_{2}^{2}$ & $6 \mathbb{R}$ & 6 \\
$\mathrm{G}_{2}$ & $\mathfrak{g}_{2} \cong \mathbb{R}^{14}$ & $T^{2}$ & $6 \mathbb{C}$ & 10 \\
\hline
\end{tabular}

TABLE III. Isotropy representations $\left(G_{2}, V_{2}\right)$ of real type irreducible isoparametric submanifolds of codimension 2 .

Finally, if $c\left(\rho_{2}\right)=1$, then we recall $\rho_{2}$ if of real type and refer to the classification to see that the outcome $\rho$ is either polar with cohomogeneity $2\left(G_{2}=\mathrm{SO}(n), \mathrm{G}_{2}\right.$ or $\operatorname{Spin}(7))$ or has cohomogeneity $3\left(G_{2}=\operatorname{Spin}(9)\right.$ or $\left.\operatorname{Sp}(n) \operatorname{Sp}(1)(n \geq 2)\right)$.

3.1.2. We address the case in which $\rho_{1}=\mathrm{SO}(3)$. Formula (1.5) gives

$$
\begin{aligned}
c(\rho) & =c\left(\rho_{2}\right)+c\left(\mathrm{SO}(2),\left[c\left(\rho_{2}\right)-1\right] \mathbb{R}^{2}\right)+c\left(H_{2}, 2 U_{2}\right) \\
& =3 c\left(\rho_{2}\right)-3+c\left(H_{2}, 2 U_{2}\right) .
\end{aligned}
$$

We immediately get $c\left(\rho_{2}\right) \leq 3$. If $c\left(\rho_{2}\right)=3$ then $c\left(H_{2}, 2 U_{2}\right)=2$, which is impossible by classification. If $c\left(\rho_{2}\right)=2$ then $c\left(H_{2}, 2 U_{2}\right) \leq 5$ implying $c\left(H_{2}, U_{2}\right) \leq 2$ which gives $c\left(H_{2}, 2 U_{2}\right)>5$, by classification. It follows that $c\left(\rho_{2}\right)=1$. We get two polar representations with $c=3\left(\rho_{2}=\mathrm{SO}(n)\right.$ or $\left.\operatorname{Spin}(7)\right)$, one representation with $c=4$ $\left(\rho_{2}=\mathrm{G}_{2}\right)$, a new example, namely, $\mathrm{SO}(3) \otimes \operatorname{Sp}(n) \operatorname{Sp}(1)(n \geq 2)$ with $c=8$, and $\mathrm{SO}(3) \otimes \operatorname{Spin}(9)$ with $c=9$.

3.1.3. For a representation $\rho_{1} \subset \mathrm{SO}(n), n \geq 3$, we have $c\left(\rho_{1} \otimes_{\mathbb{R}} \rho_{2}\right) \geq c\left(\mathrm{SO}(3) \otimes \rho_{2}\right)$ and, thus, $\rho_{2}$ is of cohomogeneity one as follows from 3.1.2. In fact, we only need to deal with real tensor representations where both factors are of cohomogeneity one and, more precisely, these are exceptional or one of $\mathrm{SO}(n), \mathrm{SU}(n), \mathrm{U}(n)$, or $\mathrm{Sp}(1) \mathrm{Sp}(2)$. Again, we rely on Lemma 2.5 for the computation of the exact cohomogeneity in most cases. For $\rho_{1}=\mathrm{SO}(n), n \geq 4$, we have $c\left(\mathrm{SO}(n) \otimes_{\mathbb{R}} \mathrm{U}(2)\right)=6$ 
and $c\left(\mathrm{SO}(n) \otimes_{\mathbb{R}} \mathrm{SU}(2)\right)=7$. Among the exceptional cohomogeneity one representations as one of the factors we retrieve $c\left(\mathrm{SO}(4) \otimes_{\mathbb{R}} \mathrm{G}_{2}\right)=8, c\left(\mathrm{SO}(5) \otimes_{\mathbb{R}} \mathrm{G}_{2}\right) \geq 11$, $c\left(\mathrm{SO}(4) \otimes_{\mathbb{R}} \operatorname{Spin}(7)\right)=5$ and $c\left(\mathrm{SO}(5) \otimes_{\mathbb{R}} \mathrm{Spin}(7)\right) \geq 9$. Moreover, we consider subrepresentations of the previous when the cohomogeneity is smaller than 9 . We discard $c\left(\mathrm{SU}(2) \otimes_{\mathbb{R}} \mathrm{G}_{2}\right) \geq c\left(\mathrm{U}(2) \otimes_{\mathbb{R}} \mathrm{G}_{2}\right) \geq 10$, and get $c\left(\mathrm{SU}(2) \otimes_{\mathbb{R}} \operatorname{Spin}(7)\right)=8$ and $c\left(\mathrm{U}(2) \otimes_{\mathbb{R}} \operatorname{Spin}(7)\right)=7$.

3.2. Quaternionic tensors. Let us consider the tensor over the quaternionic algebra of two simple Lie group quaternionic type representations.

3.2.1. We begin assuming that $\rho_{1}$ is the standard $\operatorname{Sp}(1)$-representation on $\mathbb{H}$ giving the quaternionic structure.

Let $\rho_{2}$ be $\left(\operatorname{Sp}(1), \mathbb{H}^{n}\right), n \geq 1$. The initial cases $n=1,2$ give small values of $c$. In turn, for $n \geq 4$, we have $c(\rho) \geq 4 . n-6 \geq 10$. We are left with the case $n=3$ so that $\rho_{2}=\left(\mathrm{Sp}(1), \mathbb{H}^{3}\right) \cong\left(\mathrm{SU}(2), S^{5}\left(\mathbb{C}^{2}\right)\right)$. This gives rise to $\left(\mathrm{Sp}(1) \times \mathrm{Sp}(1), \mathbb{H} \otimes_{\mathbb{H}} \mathbb{H}^{3}\right)$ which is of cohomogeneity 6 .

Assume now that the second factor is given by a bigger group, so that its dimension is at least 8 . In this case, we have

$$
2 \cdot \operatorname{dim}(G) \geq \operatorname{dim}(G)+11 \geq \operatorname{dim}(V),
$$

and the representation is either polar or, else, belongs to a finite list, as stated in [Kol03, Proposition II]. Among the non-polar cases we retrieve a cohomogeneity 6 representation given by $\left(\operatorname{Sp}(1) \otimes_{\mathbb{H}} \operatorname{Spin}(11), \mathbb{H}^{16}\right)$.

3.2.2. Consider the case where $\rho_{1}$ correspondst to $\mathrm{Sp}(1)$ acting in a non-standard fashion, i.e., $\rho_{1}=\left(\operatorname{Sp}(1), \mathbb{H}^{m}\right), m \geq 2$. Then $\rho_{1} \otimes \rho_{2} \subset \operatorname{Sp}(m) \otimes \operatorname{Sp}(n)$ with both $m, n \geq 2$. If $m \geq 3$ then

$$
c\left(\mathrm{Sp}(1) \times \mathrm{Sp}(n), \mathbb{H}^{m} \otimes \mathbb{H}^{n}\right) \geq c\left(\mathrm{Sp}(n), m . \mathbb{H}^{n}\right)-3 \geq 12 .
$$

Hence we may only admit $m=2$ and $n \geq 2$. More generally,

$$
c\left(\mathrm{Sp}(1) \times G_{2}, \mathbb{H}^{2} \otimes V_{2}\right) \geq c\left(G_{2}, 2 . V_{2}\right)-3 \geq 2 . c\left(G_{2}, V_{2}\right)-2 .
$$

Our bound on the cohomogeneity then imposes $c\left(G_{2}, V_{2}\right) \leq 5$. It is easy to consider all such possible $\rho_{2}$ since they correspond to simple Lie group representations of quaternionic type. We are thus led to a degree 4 representation of $\mathrm{Sp}(1)$ or else the standard cohomogeneity one action of $\operatorname{Sp}(n)$. The former induces a representation whose cohomogeneity is at least 10 while the latter gives a family $\left(\mathrm{Sp}(1) \times \mathrm{Sp}(n), \mathbb{H}^{2} \otimes_{\mathbb{H}} \mathbb{H}^{n}\right)$ of cohomogeneity 3 representations.

3.2.3. We still need to consider the case where both factors are given by a bigger group than $\mathrm{Sp}(1)$. Using the Monotonicity Lemma it suffices to exclude cases of the form $\operatorname{Sp}(2) \otimes \rho_{2}$. We may bound the cohomogeneity from below as

$$
c\left(\mathrm{Sp}(2) \otimes G_{2}, \mathbb{H}^{2} \otimes_{\mathbb{H}} V_{2}\right) \geq 2 . c\left(G_{2}, V_{2}\right)+1-10 .
$$

Therefore, if $c(\rho) \leq 8$, we have $c\left(\rho_{2}\right) \leq 8$. Then either $\rho_{2}$ is $\operatorname{Sp}(n)$, of cohomogeneity one, or else there are 4 such representations which are discarded by a basic dimensional bound. The argument is analogous for the first factor, thus leading to $\rho=\mathrm{Sp}(m) \otimes_{\mathbb{H}} \mathrm{Sp}(n)$ which gives a polar family. 
3.3. Complex tensors. In dealing with the remaining cases, we shall retrieve the real representations underlying complex tensor product representations. We now have a representation induced by restriction of $\mathbf{U}\left(n_{1}\right) \otimes_{\mathbb{C}} \mathrm{SU}\left(n_{2}\right) \otimes_{\mathbb{C}} \cdots \otimes_{\mathbb{C}} \mathrm{SU}\left(n_{k}\right)$ according to the number "k" of simple factors. In fact, we may have at most two simple factors since assuming otherwise leads to a subrepresentation of $\mathrm{SU}(m) \otimes_{\mathbb{C}}$ $\mathrm{SU}(n) \otimes_{\mathbb{C}} \mathrm{U}(p)$ whose cohomogeneity is at least 16 , from a natural bound for the initial case $(m, n, p)=(2,3,3)$.

3.3.1. Let us consider the case of a simple Lie group representation $\rho_{1}$ extended by a circle factor, so that $6 \leq c\left(\rho_{1}\right) \leq 9$. We are looking for the non-polar examples and, thus, may assume that the simple factor itself is not polar (as observed in GL14]). We obtain two families of examples, first those of cohomogeneity 6 given by: $\left(\mathrm{U}(6), \Lambda^{3} \mathbb{C}^{6}\right),\left(\mathrm{U}(1) \operatorname{Sp}(3), \Lambda^{3} \mathbb{C}^{6} \ominus \mathbb{C}\right),\left(\mathrm{U}(1) \operatorname{Spin}(12), \mathbb{C}^{32}\right)$ and $\left(\mathrm{U}(1) \mathrm{E}_{7}, \mathbb{C}^{56}\right)$, and those of cohomogeneity 8 corresponding to $\left(\mathrm{U}(1) \operatorname{Spin}(11), \mathbb{C}^{32}\right)$, and $\left(\mathrm{U}(1) \operatorname{Sp}(1), \mathbb{C}^{6} \cong S^{5}\left(\mathbb{C}^{2}\right)\right)$.

These examples share structural similarities, lying in-between the representations given by restriction to the simple factor, $\rho_{1}$, and the extension to $\operatorname{Sp}(1) \otimes_{\mathbb{H}} \rho_{1}$, which corresponds to an s-representation in the cases at hand. Furthermore, their cohomogeneities are known and differ by 3 , implying that the "middle" representations cannot be orbit equivalent to $\rho_{1}$. Thus, the cohomogeneity of the previous two families, of the form $\mathrm{U}(1) \otimes \rho_{1}$, equals $c\left(\rho_{1}\right)-1$.

We have made use of the fact that the quaternionic tensor product of a given quaternionic type representation $\rho_{1}$ with the standard $(\operatorname{Sp}(1), \mathbb{H})$ restricted to a circle $\mathrm{U}(1) \subset \mathrm{Sp}(1)$ gives a representation that is equivariantly isomorphic to that of the complex tensor of $\rho_{1}$ with the standard $(\mathrm{U}(1), \mathbb{C})$.

3.3.2. Let $\rho$ be $\rho_{1} \otimes \rho_{2} \subseteq \mathrm{U}(m) \otimes_{\mathbb{C}} \mathrm{SU}(n)$, with $m \geq 2$. Assume first that $\rho_{1}=\left(\mathrm{U}(2), \mathbb{C}^{2}\right)$ so that $c\left(\rho_{1} \otimes \rho_{2}\right) \geq 2 . c\left(\rho_{2}\right)-3$. The second factor is then given by a complex representation of a simple Lie group of cohomogeneity at most 5 , which is necessarily of complex type (after Remark 2.3). The tensors with such representations can be discarded using that:

$$
c\left(\mathrm{U}(2) \otimes \rho_{2}\right) \geq 2 \cdot \operatorname{dim}_{\mathbb{R}}\left(V_{2}\right)-4-\operatorname{dim}\left(G_{2}\right),
$$

unless they have cohomogeneity one. For the latter, given all the restrictions, we retrieve the polar $\mathrm{U}(m) \times \mathrm{SU}(n)$ complex-Grassmannian s-representations. If the first factor was $\rho_{1}=\left(\mathrm{SU}(2), \mathbb{C}^{2}\right)$ the cohomogeneity would only increase.

3.3.3. For $m \geq 3$ we have that $c\left(\rho_{1} \otimes \rho_{2}\right) \geq c\left(\mathrm{U}(m) \otimes \rho_{2}\right) \geq c\left(\mathrm{U}(2) \otimes \rho_{2}\right)$ and, therefore, the second factor is also of cohomogeneity one, by the previous case. In fact, both factors need to be of cohomogeneity one, thus leading to $\mathrm{U}(m) \otimes \mathrm{Sp}(n)$, $\mathrm{SU}(m) \otimes \mathrm{Sp}(n)$ and $\mathrm{SU}(m) \otimes \mathrm{SU}(n)$. We compute their cohomogeneity with the aid of Lemma 2.5

\section{REFERENCES}

[AA93] A. Alekseevsky and D. Alekseevsky. Asystatic G-manifolds. In Differential geometry and topology (Alghero, 1992), pages 1-22. World Sci. Publ., River Edge, NJ, 1993.

[BCO03] J. Berndt, S. Console, and C. Olmos. Submanifolds and holonomy. Number 434 in Research Notes in Mathematics. Chapman \& Hall/CRC, Boca Raton, 2003.

[Bor49] A. Borel. Some remarks about lie groups transitive on spheres and tori. Bull. Amer. Math. Soc., 55:580-587, 1949. 


\begin{tabular}{|l|l|l|}
\hline$G$ & $V$ & Condition \\
\hline \hline $\mathrm{SO}(3)$ & $\mathbb{R}^{9}$ & - \\
$\mathrm{SU}(5)$ & $S^{2} \mathbb{C}^{5}$ & - \\
$\mathrm{SU}(10)$ & $\Lambda^{2} \mathbb{C}^{10}$ & - \\
$\mathrm{U}(1) \times \mathrm{SO}(3)$ & $\mathbb{C}^{5}$ & - \\
$\mathrm{U}(1) \times \mathrm{Sp}(3)$ & $\Lambda^{2} \mathbb{C}^{6} \ominus \mathbb{C}$ & - \\
$\mathrm{U}(1) \times \mathrm{Sp}(3)$ & $\Lambda^{3} \mathbb{C}^{6} \ominus \mathbb{C}^{6}$ & - \\
$\mathrm{U}(1) \times \mathrm{SU}(6)$ & $\Lambda^{3} \mathbb{C}^{6}$ & - \\
$\mathrm{U}(1) \times \mathrm{Spin}(12)$ & $\mathbb{C}^{32}$ & - \\
$\mathrm{U}(1) \times \mathrm{E}_{7}$ & $\mathbb{C}^{56}$ & - \\
$\mathrm{SO}(3) \times \mathrm{SU}(2)$ & $\mathbb{R}^{3} \otimes_{\mathbb{R}} \mathbb{C}^{2}$ & - \\
$\mathrm{SO}(3) \times \mathrm{SU}(n)$ & $\mathbb{R}^{3} \otimes_{\mathbb{R}} \mathbb{C}^{n}$ & $n \geq 4$ \\
$\mathrm{SO}(3) \times \mathrm{U}(n)$ & $\mathbb{R}^{3} \otimes_{\mathbb{R}} \mathbb{C}^{n}$ & $n \geq 3$ \\
$\mathrm{SO}(n) \times \mathrm{U}(2)$ & $\mathbb{R}^{n} \otimes_{\mathbb{R}} \mathbb{C}^{2}$ & $n \geq 4$ \\
$\mathrm{Sp}(1) \times \mathrm{Sp}(1)$ & $\mathbb{H} \otimes_{\mathbb{H}} \mathbb{H}^{3}$ & - \\
$\mathrm{Sp}(1) \times \mathrm{Spin}(11)$ & $\mathbb{H}_{\mathbb{H}} \otimes_{\mathbb{H}} \mathbb{H}^{16}$ & - \\
$\mathrm{SU}(5) \times \mathrm{SU}(5)$ & $\mathbb{C}^{5} \otimes_{\mathbb{C}} \mathbb{C}^{5}$ & - \\
$\mathrm{U}(3) \times \mathrm{Sp}(n)$ & $\mathbb{C}^{3} \otimes_{\mathbb{C}} \mathbb{H}^{n}$ & $n \geq 3$ \\
$\mathrm{U}(n) \times \mathrm{Sp}(2)$ & $\mathbb{C}^{n} \otimes_{\mathbb{C}} \mathbb{H}^{2}$ & $n \geq 4$ \\
$\mathrm{SU}(n) \times \mathrm{Sp}(2)$ & $\mathbb{C}^{n} \otimes_{\mathbb{C}} \mathbb{H}^{2}$ & $n=3, n \geq 5$ \\
\hline
\end{tabular}

TABLE IV. Non-polar irreducible representations of cohomogeneity 6.

\begin{tabular}{|l|l|l|}
\hline$G$ & $V$ & Condition \\
\hline \hline $\mathrm{Sp}(3)$ & $\Lambda^{3} \mathbb{C}^{6} \ominus \mathbb{C}^{6}$ & - \\
$\mathrm{SU}(6)$ & $\Lambda^{3} \mathbb{C}^{6}$ & - \\
$\mathrm{SU}(6)$ & $S^{2} \mathbb{C}^{6}$ & - \\
$\mathrm{SU}(12)$ & $\Lambda^{2} \mathbb{C}^{12}$ & - \\
$\mathrm{Spin}(12)$ & $\mathbb{C}^{32}$ & - \\
$\mathrm{E}_{7}$ & $\mathbb{C}^{56}$ & - \\
$\mathrm{U}(1) \times \mathrm{SU}(3)$ & $\mathfrak{s u}(3)^{\mathbb{C}}$ & - \\
$\mathrm{U}(1) \times \mathrm{F}_{4}$ & $\mathbb{C}^{26}$ & - \\
$\mathrm{U}(2) \times \mathrm{Spin}(7)$ & $\mathbb{C}^{2} \otimes_{\mathbb{R}} \mathbb{R}^{8}$ & - \\
$\mathrm{SO}(3) \times \mathrm{SU}(3)$ & $\mathbb{R}^{3} \otimes_{\mathbb{R}} \mathbb{C}^{3}$ & - \\
$\mathrm{SO}(n) \times \mathrm{SU}(2)$ & $\mathbb{R}^{n} \otimes_{\mathbb{R}} \mathbb{C}^{2}$ & $n \geq 4$ \\
$\mathrm{SU}(6) \times \mathrm{SU}(6)$ & $\mathbb{C}^{6} \otimes_{\mathbb{C}} \mathbb{C}^{6}$ & - \\
$\mathrm{SU}(3) \times \mathrm{Sp}(n)$ & $\mathbb{C}^{3} \otimes_{\mathbb{C}} \mathbb{H}^{n}$ & $n \geq 3$ \\
$\mathrm{SU}(4) \times \mathrm{Sp}(2)$ & $\mathbb{C}^{4} \otimes_{\mathbb{C}} \mathbb{H}^{2}$ & - \\
\hline
\end{tabular}

TABLE V. Non-polar irreducible representations of cohomogeneity 7.

[Dad85] J. Dadok. Polar coordinates induced by actions of compact Lie groups. Trans. Amer. Math. Soc., 288:125-137, 1985.

[GG] C. Gorodski and F. J. Gozzi. Representations with $\mathrm{Sp}(1)^{\mathrm{k}}$-reductions and quaternionkaehler symmetric spaces.

[GK16] Claudio Gorodski and Andreas Kollross. Some remarks on polar actions. Ann. Global Anal. Geom., 49(1):43-58, 2016. 


\begin{tabular}{|l|l|}
\hline$G$ & $V$ \\
\hline \hline $\mathrm{SO}(3)$ & $\mathbb{R}^{11}$ \\
$\mathrm{SU}(14)$ & $\Lambda^{2} \mathbb{C}^{14}$ \\
$\mathrm{SU}(7)$ & $S^{2} \mathbb{C}^{7}$ \\
$\mathrm{U}(1) \times \mathrm{SU}(2)$ & $\mathbb{H}^{3}$ \\
$\mathrm{U}(1) \times \operatorname{Spin}(11)$ & $\mathbb{H}^{16}$ \\
$\mathrm{SU}(7) \times \mathrm{SU}(7)$ & $\mathbb{C}^{7} \otimes_{\mathbb{C}} \mathbb{C}^{7}$ \\
$\mathrm{SU}(2) \times \mathrm{Spin}(7)$ & $\mathbb{C}^{2} \otimes_{\mathbb{R}} \mathbb{R}^{8}$ \\
$\mathrm{SO}(4) \times \mathrm{G}_{2}$ & $\mathbb{R}^{4} \otimes_{\mathbb{R}} \mathbb{R}^{7}$ \\
$\mathrm{SO}(3) \times \mathrm{Sp}(1) \mathrm{Sp}(2)$ & $\mathbb{R}^{3} \otimes_{\mathbb{R}} \mathbb{H}^{2}$ \\
\hline
\end{tabular}

TABLE VI. Non-polar irreducible representations of cohomogeneity 8.

[GL14] C. Gorodski and A. Lytchak. On orbit spaces of representations of compact Lie groups. J. reine angew. Math., 691:61-100, 2014.

[GL15a] C. Gorodski and A. Lytchak. Isometric actions on spheres with an orbifold quotient. Math. Ann., pages 1-27, 2015. doi:10.1007/s00208-015-1304-y.

[GL15b] C. Gorodski and A. Lytchak. Representations whose minimal reduction has a toric identity component. Proc. Amer. Math. Soc., 143:379-386, 2015.

[GT00] C. Gorodski and G. Thorbergsson. Representations of compact Lie groups and the osculating spaces of their orbits. Preprint, Univ. of Cologne, (also E-print math. DG/0203196), 2000.

[GT03] C. Gorodski and G. Thorbergsson. The classification of taut irreducible representations. J. reine angew. Math., 555:187-235, 2003.

[GZ12] Karsten Grove and Wolfgang Ziller. Polar manifolds and actions. J. Fixed Point Theory Appl., 11(2):279-313, 2012.

[HH70] Wu-chung Hsiang and Wu-yi Hsiang. Differentiable actions of compact connected classical groups. II. Ann. of Math. (2), 92:189-223, 1970.

[HL71] W.-Y. Hsiang and H. B. Lawson Jr. Minimal submanifolds of low cohomogeneity. J. Differential Geom., 5:1-38, 1971.

[Hsi67] W-Y. Hsiang. On the principal orbit type and P. A. Smith theory of $s u(p)$ actions. Topology, 6(1):123-135, 1967.

[Kol02] Andreas Kollross. A classification of hyperpolar and cohomogeneity one actions. Trans. Amer. Math. Soc., 354(2):571-612 (electronic), 2002.

[Kol03] A. Kollross. Low cohomogeneity representations and orbit maximal actions. Ann. Global Anal. Geom., 23:93-100, 2003.

[MS43] Deane Montgomery and Hans Samelson. Transformation groups of spheres. Ann. of Math. (2), 44:454-470, 1943.

[Oni04] A. L. Onishchik. Lectures on real semisimple Lie algebras and their representations. ESI Lectures in Mathematics and Physics. European Mathematical Society, Zürich, 2004.

[Str94] E. Straume. On the invariant theory and geometry of compact linear groups of cohomogeneity $\leq$ 3. Diff. Geom. and its Appl., 4:1-23, 1994.

Instituto de Matemática e Estatística, Universidade de São Paulo, Rua do Matão, 1010, São Paulo, SP 05508-090, Brazil

E-mail address: fj.gozzi@gmail.com 